\title{
BEOBACHTUNGEN ZUR SOLIFLUKTION IN SCHWEDISCH LAPPLAND
}

\author{
RÜDIger German
}

Mit 4 Photos

Anläßlich einer kurzen Studienreise durch Schweden hatte der Verfasser Gelegenheit, während einiger weniger Tage in der Gegend von Abisko am Torneträsk (Schwedisch Lappland) u.a. Solifluktionserscheinungen zu beobachten. Leider gestattete die fortgeschrittene Jahreszeit nicht mehr, Solifluktionserscheinungen auf den höchsten Bergen zu beobachten, da am Anfang über $1500 \mathrm{~m} \mathrm{NN}$, am Ende des Aufenthaltes über $1200 \mathrm{~m}$ NN schon Schnee lag. Die Solifluktionserscheinungen treten, wie schon C. RathJENS und H. v. Wissmann (1929) erkannten, in Lappland erst oberhalb der Baumgrenze in deutlicher Ausbildung auf. Darunter macht die dichte Bodenvegetation das Erkennen fast uninöglich.

Aus der Umgebung von Abisko sind bisher folgende Solifluktionserscheinungen bekannt geworden :

1. Schutt-Tropfen (RathJens und Wissmann 1929),

$\left.\begin{array}{l}\text { 2. Fließerdeterrassen } \\ \text { 3. Fließerdewülste }\end{array}\right\}$

(SJÖGREN 1909)

Verfasser konnte darüber hinaus von den bisher aus anderen Teilen Skandinaviens bekannten und bei C. Troll (1949, S. 620) zusammengestellten Struktur- und Solifluktionsböden folgende Arten beobachten:
4. Steinströme
5. Steinnetze und Stein ringe
6. Schuttstreifenböden.

\section{SCHUTT-TROPFEN}

Sie wurden von Rathjens und v. Wissmann (1929, S. 124) als Ansammlungen groben Schuttes beschrieben, der «mit Blöcken bis zu 0,50 m Durchmesser in Tropfenform angeordnet», talab wandert. «Am Stirnende des Tropfens, wo die größeren Blöcke liegen, ist der Boden mitsamt der Vegetationsdecke oft aufgewulstet, ein Beweis, daß der Schuttropfen in einer Abwärtsbewegung begriffen ist. Am oberen Ende des Schuttropfens befindet sich oft eine flache, dreiseitig geschlossene Mulde, aus der augenscheinlich das Schuttmaterial des Tropfens stammt. Am Boden dieser Mulde ist es feucht und sumpfig. In einzelnen Fällen sickert am Grunde sogar Wasser hervor, das aber gleich wieder im Schutt verschwindet.» Diese Beobachtung von RathJEns und v. Wissmann konnten an anderer Stelle in der Umgebung des Torneträsk wiedergefunden und bestätigt werden.

a) Die Bildung der Schutt-T'ropfen. Ergänzend zu diesen Beschreibungen möchte Verfasser die Entstehung der Mulde, sowie die Anordnung und Wanderung des Schutt-Tropfens folgendermaßen erklären: Die Mulde am oberen Rand des SchuttTropfens konnte gegenüber der Umgebung als ein Ort bevorzugter Wasseransammlung, als eine Art Quellnische erkannt werden. In ihr tritt bei der Schneeschmelze durch Klüfte oder Gesteinslagerung begünstigt, etwas Wasser an die Oberfläche, das bei Frost gefriert. Durch die Wirkung des Spaltenfrostes zerfällt das Anstehende in größere und kleinere Gesteinsteilchen, die beim Tauen durch Solifluktion in Bewegung geraten. Die kleineren Teile werden durch die weitere Verwitterung rascher zerkleinert als die Gesteinsplatten und bilden das Gleitmittel für die größeren Steine. Das Wasser, das in den Hangnischen am oberen Ende des Tropfens in Zeiten der Schneeschmelze oder bei Niederschlag sichtbar wird, versickert sofort zwischen den hangabwärts sich anschließenden Gesteinsplatten und im Schutt. Darunter fließt das Wasser unsichtbar weiter. Fällt einmal viel Wasser an, so kann dieses am Ende des Tropfens wieder austreten und bildet ein kleines Rinnsal. 


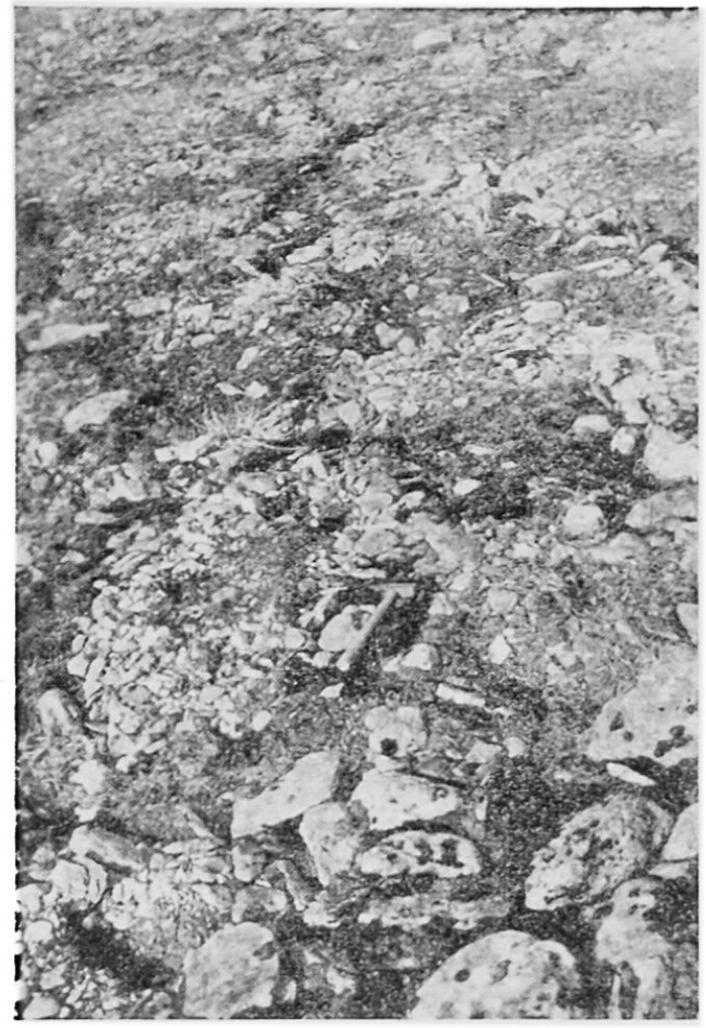

Abb. 1 Steinringe am Laktatjokko (Hammer zum Vergleich).

b) Die Form der Schutt-Tropfen. Die charakteristische Form dieser Schuttbildung scheint mir nicht streng an die hier beschriebene Art der Schutt-Tropfen gebunden zu sein. Wir finden diese Tropfenform gelegentlich auch bei den Stein- und Schutt-Strömen, also bei Schuttbildungen, deren Anfang an keine Nische oder Mulde gebunden ist (vgl. Nr. 4). Wie überhaupt auf Grund der Strömungserscheinungen zu erwarten ist, muß es sich hierbei um eine allgemeinere Erscheinung handeln, die bei allen hier beschriebenen Schuttbildungen des Solifluktionsbereiches auftreten kann.

Bei den Schutt-Tropfen wird die charakteristische Form dadurch erzielt, da $\beta$ der anfänglich schmale Tropfen, der meist in einer Mulde liegt, nunmehr in eine flachere Senke mündet oder einen breiten Hang hinabfließt. Durch Ausflachung der Mulde konnte sich der Tropfen verbreitern. Infolge des hier - senkrecht zur Fließrichtung gemessenen - flachen Hangwinkels als in der Stamm-Mulde ist die Ausdehnung des Tropfens in die Breite verständlich. Während er zuerst ähnlich wie Flüsse oder Gletscher in einem schmalen Kerb bzw. Trogtal eingeengt liegt, vermag der SchuttTropfen sich im weniger eingemuldeten Gelände auszubreiten, ähnlich wie dies bei Flüssen in einem Flachland oder bei Gletschern im Gebirgsvorland möglich ist. Darüberhinaus ist die Breitenausdehnung auch noch von der Fließgeschwindigkeit und der Gesteinsmenge abhängig, die ihrerseits wieder durch den Hangwinkel in Fließrichtung und durch die Zähigkeit des Schuttes bedingt sind.

Bei den Stein- und Schuttströmen kann die Tropfenform auf die gleiche Weise entstehen. Bei diesen tritt sie jedoch im Beobachtungsgebiet viel seltener auf. Die Schuttströme werden ja in den Gipfelregionen auf breiten Flächen gebildet und fließen meist in schmäleren, erosiv gebildeten Rinnen hangab. Dadurch, daß keine Ausflachung der Mulde, sondern im Gegenteil eine Verengung eintritt, sind sie in eine schmälere Form gezwungen und können sich nicht ausbreiten. Im Prinzip ist es jedoch möglich, daß sich beide Erscheinungen - Schuttströme und Schutt-Tropfen - in ihrer äußeren Form einander weitgehend annähern.

Wir sehen hieraus, daß auch die Form der Schuttbildungen von den mechanischen Strömungs- und Untergrundverhältnissen abhängig ist und daß die formenmäßige Vielheit sich mit Hilfe physikalisch-mechanischer Grundlagen auf einfache Weise erklären läßt. Aus diesem Grunde hat eine genetische Klassifikation dieser Bildungen in erster Linie von diesen Grundtatsachen auszugehen. - Bei weiteren Beobachtungen muß also stets die Hangneigung und das umgebende Relief mit dem Hangwinkel senkrecht zur Fließrichtung erfaßt werden, um an Hand von zahlreichen Beispielen einen groben Anhalt für die Abhängikeit der Fließgeschwindigkeit und der Schuttform vom Gelände zu erhalten. Es sei allerdings gleich darauf hingewiesen, daß außerdem noch zahlreiche andere Faktoren mitspielen, die sich nicht genau bestimmen lassen, wie $z$. B. die Zähigkeit des Schuttstromes, die von den Wasser- und Temperaturverhältnissen abhängig ist und zeitlich stark schwankt. 


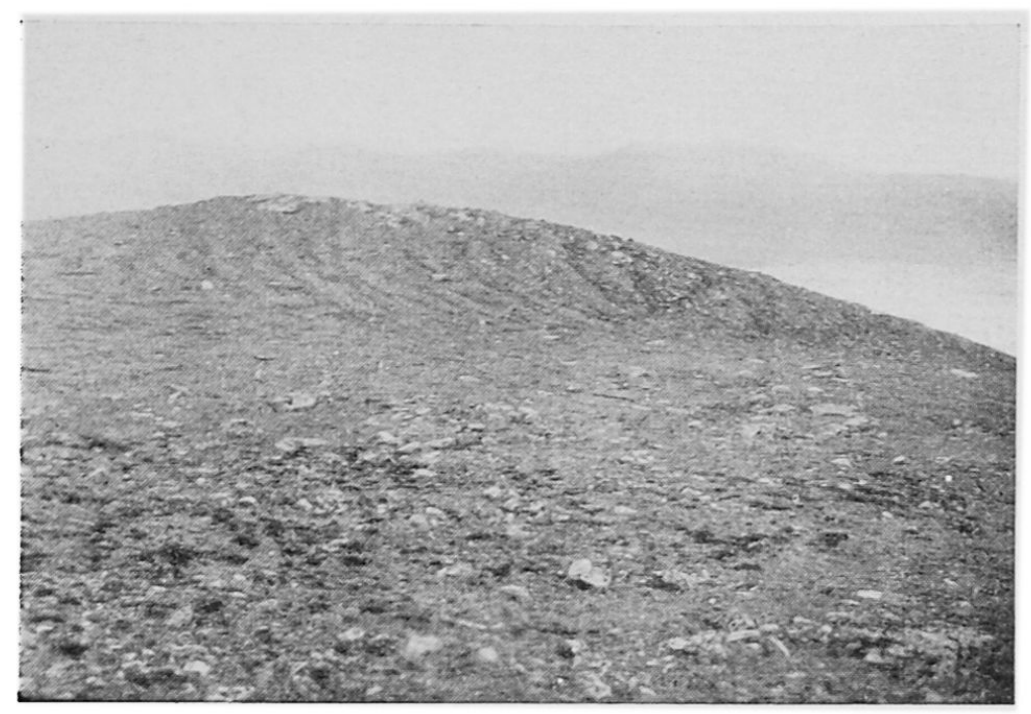

Abb. 2

Schuttstreifenböden auf dem Nuolja, in eine Mulde einbiegend (Südexposition).

Photo German

c) Die Länge der Schutt-Tropfen. Die bisher von Rathunens und v. Wissmann beschriebenen Schutt-Tropfen besitzen eine Länge von 20-30 m. Verfasser schätzte bei seinen Beobachtungen auch etwa reichlich $30 \mathrm{~m}$. Da wir wissen, daß jene Gegend erst seit einigen tausend Jahren eisfrei ist (das Ende des Finnglazials liegt etwa 8000 Jahre zurück), können wir versuchen, die ungefähre Wanderungsgeschwindigkeit der Schutt-Tropfen am Torneträsk zu bestimmen. Bei einer einfachen Division der Maßzahl der Länge des Tropfens durch die Zahl von 8000 Jahren ergeben sich etwa 30 $40 \mathrm{~cm}$ in 100 Jahren. Dieser Wert muß aber noch korrigiert werden, da ja die Tropfenform ein ungleichmäßiges Wandern auf der ganzen Erstreckung des Tropfens anzeigt. Im oberen Teil wäre daher entsprechend den mechanischen Strömungsgesetzen (bei geringer Strombreite) mit einer wesentlich größeren und im unteren Teil (bei größerer Strombreite) mit einer wesentlichen geringeren Wanderungsgeschwindigkeit zu rechnen. Außerdem ist zu berücksichtigen, daß bei zunehmendem Größerwerden der Stamm-Mulde auch mehr Schutt gebildet werden kann. Bei dem oben errechneten Wert handelt es sich also um eine sehr grobe Mittelwertbildung.

Ein weiteres anschauliches Beispiel des Bodenfließens konnte am Låktatjåkko unterhalb einer kompakten Gesteinsbank beobachtet werden, die hangab in viele große Gesteinsplatten aufgespalten war und auseinanderfloß. Die einzelnen Platten wurden im Laufe ihres Weges immer kleiner. Dafür nahm der kleine Verwitterungsschutt dazwischen zu. Die Erstreckung der ganzen Fließerscheinung betrug wieder etwa 25-30 m.

In der gleichen Größenordnung liegt übrigens auch die Länge des Schuttstreifenbodens in der Abb. 2, so daß wohl auch bei dieser Solifluktionserscheinung auf ähnliche Fließgeschwindigkeit geschlossen werden darf.

d) Die Stirn der Schutt-Tropfen. Der vordere Rand der Tropfen ist, wie schon Rathjens und v. Wissmann berichten, aufgewulstet. Eine genaue Analyse dieser Erscheinung ist besonders wichtig. Bei dem Hangabfließen des Schutt-Tropfens und des Schuttstromes wird schließlich ein Vegetationspolster oder die geschlossene Rasenfläche erreicht. Dadurch erfährt der Strom einen Widerstand, seine Fortbewegung ist gehemmt. Die Wurzeln der Pflanzen versuchen aber möglichst den Zusammenhang der Bodenschicht zu erhalten. In jenen Gebieten ist die Bodenschicht (mit viel Verwitterungsgrus) nur sehr dünn, namentlich an der oberen Grenze geschlossenen Pflanzenwuchses. Da diese Gebiete noch nicht sehr lange vom Eise verlassen wurden, folgt unter dieser entweder gleich der anstehende Fels oder der ebenfalls gering mächtige Verwitterungsgrus. 
Für die weitere Fortbildung des Schuttes ist die Zone zwischen der zusammengehaltenen Grasnarbe und dem kompakten Anstehenden wichtig. In ihr bildet sich der Verwitterungsgrus, der zusammen mit dem Wasser, das bis auf das Anstehende durchsickert, wieder das Gleitmittel bildet. Hier ist also die optimale Zone der Fließbewegung. Der Schuttstrom bzw. -tropfen, wie auch die Fließ-Schutt-Terrasse (s.u.) scheinen also beim Hangabfließen die Grasnarbe zu heben, unter diese hineinzugleiten und

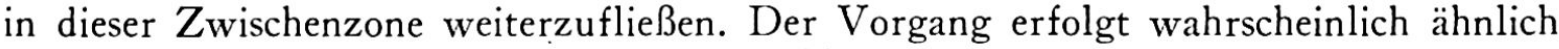
wie das Rasenwälzen (Troll 1944, S. 558/559). Der Schutt, der manchmal unter der Rasendecke vorhanden ist, wird dabei aufgearbeitet. Infolge der Reibung am Untergrund und in geringerem Maße auch am Hangenden einerseits und der Zähigkeit des Fließmittels andererseits treten bei diesen Gleitvorgängen (Schuttstrom, Schutt-Tropfen, Fließ-Schutt-Terrassen und Rasenwälzen) Faltenbildungen auf, wie dies auf Grund der mechanischen Strömungsbedingungen erwartet werden kann. Da das Hangabwandern dieser Schuttbildungen in Lappland lange Zeit dauert, hat die Vegetation Zeit, an den aufgewölbten Rändern weiter emporzuwachsen. Durch diesen Widerstand an der Stirn der Schutt-Tropfen wird deren Wanderungsgeschwindigkeit am unteren Ende weiter stark vermindert.

\section{FLIESS-SCHUTT-TERRASSEN (Fließerdterrassen)}

«Die Fließerdterrassen die auf großen Flächen des Gebirgsplateaus dem Terrain ihr Gepräge verleihen, kommen am zahlreichsten in den waldlosen Partien der Abiskoebene oder an den Abhängen der Gebirge vor, die aus Granatschimmerschiefer aufgebaut sind.» (SJöGREN 1909, S. 10). Demnach und nach eigenen Geländebeobachtungen handelt es sich allerdings weniger um «Erde» als weitgehend um Gesteinsschutt und V'erwitterungsgrus. Der Name Fließ-Schutt-Terrassen kommt daher den Tatsachen näher. Auch am Vorderrande dieser Bildung wächst die spärliche Vegetation empor und unterstreicht die bekannte Stufenform. Die horizontale Oberfläche der Stufe trägt dagegen kein Pflanzenkleid, sondern zeigt nur Schutt. Am Nuolja konnte in Nordexposition und etwa $1100 \mathrm{~m}$ NN ein breites Feld solcher Fließ-Schutt-Terrassen beobachtet werden.

\section{F L I E S S-S CHUTT-WÜ LSTE (Fließerdewülste)}

Vielfach tritt der Fließ-Schutt in weniger geschlossener Form wie in den Terrassen auf und erzeugt nur einzelne Wülste. Analog den Fließ-Schutt-Terrassen nennt man diese bisher Fließerdewülste genannten Formen nach ihrer Materialzusammensetzung kennzeichnender Fließ-Schutt-Wülste.

\section{STEINSTRÖME}

Ein Steinstrom, der in ungefähr $1100 \mathrm{~m} \mathrm{NN}$ am Slåttatjåkko liegt und sich in einer kleinen Mulde hangab bewegt, zeigt folgendes (vgl. Abb. 3): Abgesehen von einigen obenauf liegenden Steinen ordnen sich die Platten einigermaßen in der Strömungsrichtung an. In der Mitte des Stromes stehen die Platten ungefähr senkrecht, während sie sich am Rande, wo sie nicht mehr von Nachbarplatten gestützt werden, nach außen zu neigen. Damit hängt wohl auch die leichte Aufwölbung zusammen, die in der Mitte des Steinstromes zu beobachten ist.

Im Gegensatz zu den oben erwähnten Schuttropfen hängt ihre Bildung jedoch nicht mit Nischen usw. zusammen, sondern das Verwitterungsmaterial der Gipfelregion wird in diesen Strömen gesammelt und hangab transportiert. Das Gleitmaterial bildet feines Verwitterungsmaterial (Grus), das bei der Schneeschmelze mit dem leicht in die Mulde einfließenden Schmelzwasser durchsetzt wird. Auf diesem weichen Brei ist bei wiederholtem Gefrieren und Tauen ein leichter Gesteinstransport möglich. (Wegen der Form der Steinströme vgl. die oben aufgeführten Schuttropfen.) 


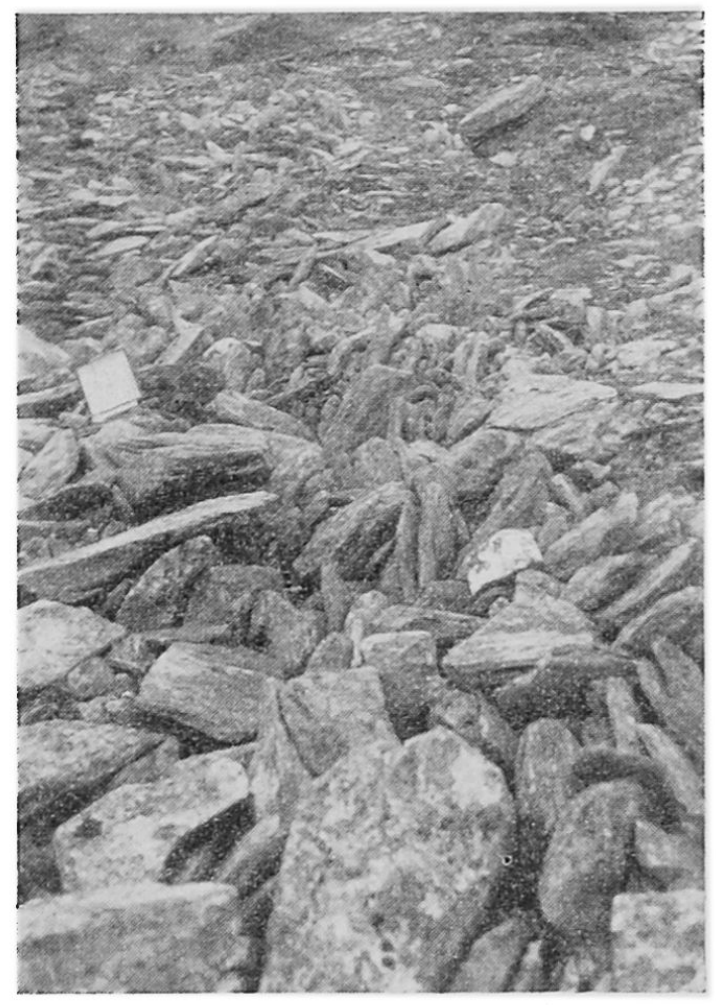

Abb. 3 Teilansicht eines Schuttstromes am Slattajakko (Kartentasche zum Vergleich). Photo German

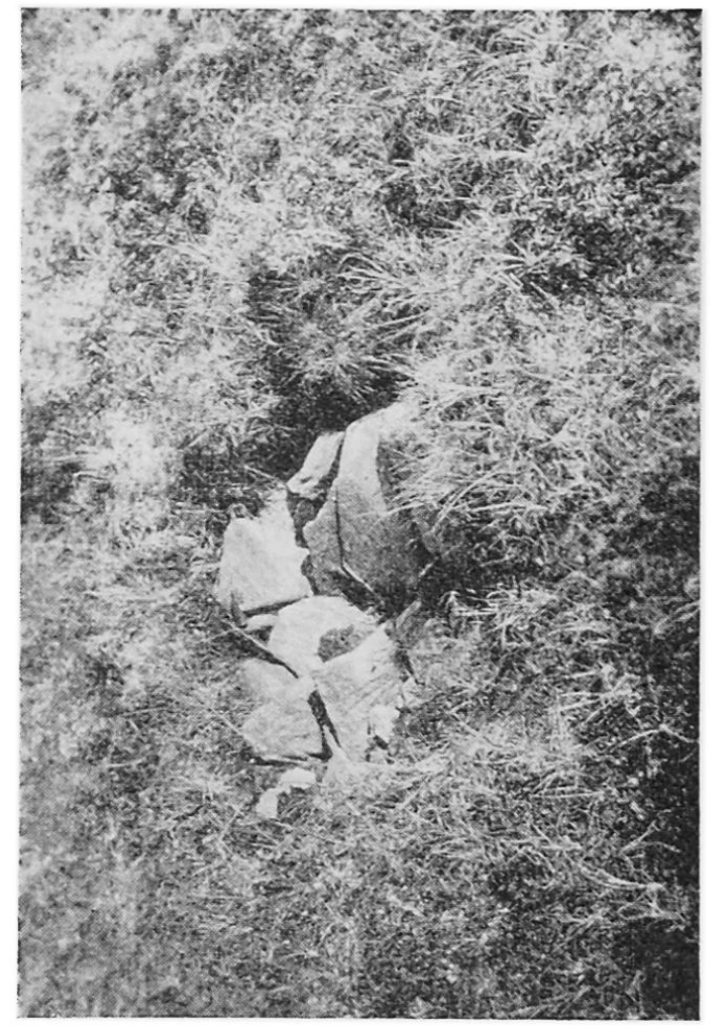

Abb. 4 Steininsel am Laktatjakko (Hammer zum Vergleich).

Photo German

\section{STEINNETZE UND STEINRINGE}

Die vom Verfasser beobachteten Steinnetze und Steinringe hatten meist einen Durchmesser von etwa $60 \mathrm{~cm}$ (vgl. Abb. 1). Sie waren nicht in Besonders schöner und gleichmäßig runder Form ausgebildet. Einzelne Vorkommen am Aufstieg zum Låktatjåkko waren in etwa $1050 \mathrm{~m}$ NN noch kleiner und erreichten als kleinsten beobachteten Durchmesser 25-30 cm (Abb. 1, linke untere Ecke).

\section{SCH UTTSTREIFENBÖDEN}

Schuttstreifenböden (Abb. 2) konnten auf der nach Norden vorspringenden Nase des Nuolja in etwa $1030 \mathrm{~m}$ NN beobachtet werden. Die Streifen verlaufen anfänglich in nord-südlicher Richtung und biegen bald, einer Mulde folgend, nach Osten ab.

\section{STEININSELN}

Außer diesen bisher schon bekannten Struktur- und Solifluktionsformen, die im allgemeinen über der Baumgrenze auftreten, konnte innerhalb der Vegetationszone nahe ihrer oberen Grenze eine weitere Bildung in großer Zahl festgestellt werden. Innerhalb der Bodenvegetation war im Gras ein Loch ausgespart, in dem sich zahlreiche Steine befanden (Abb.4). Diese Steine hatten einen größten Durchmesser von etwa $20 \mathrm{~cm}$ und stellten eine inselförmige Anreicherung von groben Steinen dar. Ich möchte daher im Gegensatz zu den Steinringen den Namen Steininseln vorschlagen.

\section{LITERATUR}

1. Rathjens, C. und Wissmann H.v.; Oberflächenformen und Eisböden in Lappland. Peterm. Mitteilungen 75, 1929, S. 120-126. - 2. SJöGREN, O.: Der Torneträsk: Morphologie und Glazialgeo- 
logie. Exkursionsführer zum XI. Internationalen Geologiekonkress in Stockholm 1910. Abdruck aus Geol. Fören. i Stockholm, Forhandl. Bd. 31, H. 6, Nov. 1909. - 3. Troll, C.: Strukturböden, Solifluktion und Frostklimate der Erde. Geolog. Rundschau 34, 1944, S. 545.

\title{
OBSERVATIONS DE PHÉNOMENES DE SOLIFLUCTION EN LAPONIE SUÉDOISE
}

\begin{abstract}
Outre les phénomènes de solifluction qui ont déjà été étudiés à Abisko, au bord du Torneträsk (Laponie suéd.), łles lentilles de débris, les terrasses et les bombements de sol fluent, on a encore noté des coulées de pierres, des sols détritiques striés, ainsi que des réseaux et des cercles de pierres. Il est discuté de la forme et de la genese de ces lentilles et, par la même occasion, de leur comportement sur le front de la formation. Sur la base de ces observations, l'auteur propose d'adopter pour les terrasses de sol fluent le nom de terrasses de débris fluents (FliessSchutt-Terrassen) et pour les bombements de sol fluent celui de bombements de débris fluents (Fliess-Schutt-Wülste).
\end{abstract}

\section{WELLEN UND ZYKLEN DER KULTUR}

\author{
BEITRAG ZUR HISTORISCHEN GEOGRAPHIE
}

\author{
EDUARD Markus
}

\begin{abstract}
Die vorliegende Abhandlung stellt einen Auszug aus einer Untersuchung dar, die der historischen Geographie der Kultur gewidmet ist. Sie erörtert eine spezielle Methode und legt Schlußfolgerungen dar, die sich aus dieser Methode ableiten lassen. Mit ihrer Hilfe versucht sie die gegenwärtige Krise zu erklären und einige Zukunftsaussichten zu äußern.
\end{abstract}

\section{METHODE}

Wesen. Die historische Geographie behandelt bestimmte Erscheinungen in der historischen Zeit und im geographischen Raum (3). Wir verfolgen zunächst hochbegabte Personen, welche die leitenden Gedanken der Kultur entwickelt haben. Ihre Werke helfen uns, sie miteinander zu vergleichen. Untersuchungen der menschlichen Begabung erleichtern unsere Arbeit. Nach Terman und Cox (9) haben Genies folgende Intelligenzquoten: Goethe 210, Pascal 195, Newton 190, Galileo 185, Descartes 180 usw. Der Forderung der historischen Geographie gemäß untersuchen wir die Verteilung der hochbegabten Personen in der Zeit und im Raume. Dabei benutzen wir spezielle Diagramme, die schaffende Persönlichkeiten und Elemente der Kultur darstellen (Abb. 1 und 2). Solche Diagramme zeigen, daß hochbegabte Personen gruppenweise auftreten - sich in einigen Perioden häufen und dadurch die Blütezeiten der Kultur versinnbildlichen.

Anwendung. Zur Erläuterung unserer Methode verfolgen wir die Verlagerung der Kultur aus der Trockenzone in den subtropischen Teil des gemäßigten Klimagürtels. Die meisten alten Kulturen sind bekanntlich in Flußtälern der 'Trockenzone entstanden - in Mesopotamien und Ägypten, im Tal des Indus und im Gebiete von Hwang-ho und Wei-ho in China, wo künstliche Bewässerung den zivilisatorischen Fortschritt des Menschen begünstigte. Im 7.Jahrhundert v. Chr. erlebte die ganze zivilisierte Welt eine tiefe politische und geistige Krise. Insbesondere wurde eine Schwächung alter Staaten der Flußoasen zwischen 700 und 600 v. Chr. festgestellt. Die Zerstörung von Babylon 689, die Zerstückelung von China 655 und der Zusammenbruch des Assyrischen Reiches in der zweiten Hälfte des 7. Jahrhunderts sind Kennzeichen dieser vor allem politischen Krise, während Ägypten unzulängliche Herrscher hatte, so daß es 671 in eine assyrische Provinz verwandelt wurde. Die großen Propheten Jesaia, Jeremia und andere, die in dieser Zeit lebten (Abb. 1), beschrieben eine religiöse Krise und eine moralische Verwilderung orientalischer Völker in Palästina, Mesopotamien und Ägypten. Die Biographie von Konfutse erzählt vom Verfall der politischen Moral in China. 